\title{
Antiarrhythmic properties of atrial pacing
}

\author{
Magdalena Klis' 1 ,A-F, Agnieszka Sławuta ${ }^{2, A-F}$, Jacek Gajek ${ }^{3, A-F}$ \\ ${ }^{1}$ Department of Cardiology, Province Specialist Hospital, Wrocław, Poland \\ 2 Department of Cardiology, Klodzko County Hospital, Poland \\ ${ }^{3}$ Department of Clinical Nursing, Wroclaw Medical University, Poland \\ A - research concept and design; $\mathrm{B}$ - collection and/or assembly of data; $\mathrm{C}$ - data analysis and interpretation; \\ $D$ - writing the article; $E$ - critical revision of the article; $F$ - final approval of article
}

\section{Address for correspondence \\ Jacek Gajek}

E-mail: jacek.gajek@interia.pl

\section{Funding sources \\ none declared}

\section{Conflict of interest}

none declared

Received on November 08, 2015

Revised on November 18, 2015

Accepted on January 19, 2016

DOI

\begin{abstract}
Bradycardia, atrial stretch and dilatation, autonomic nervous system disorders, and the presence of triggers such as atrial premature contractions, are factors which predispose a person to paroxysmal AF. Atrial pacing not only eliminates bradycardia but also prevents atrial premature contractions and dispersion of refractoriness, which are a substrate for atrial fibrillation. As the prolonged duration of atrial activation during pacing, especially from locations changing the physiological pattern of this activation (right atrium lateral wall, right atrium appendage), negatively influences both a mechanical and an electrical function of the atria, the atrial pacing site affects an atrial arrhythmogenesis. A conventional atrial lead location in the right atrium appendage causes non-physiological activation propagation, resulting in a prolongation of the activation time of both atria. This location is optimal according to a passive fixation of the atrial lead but the available contemporary active fixation leads could potentially be located in any area of the atrium. There is growing evidence of the benefit of pacing, imitating the physiological propagation of impulses within the atria. It seems that the Bachmann's bundle pacing is the best pacing site within the atria, not only positively influencing the atrial mechanical function but also best fulfilling the so-called atrial resynchronization function, in particular in patients with interatrial conduction delay. It can be effectively achieved using only one atrial electrode, and the slight shortening of atrioventricular conduction provides an additional benefit of this atrial pacing site.
\end{abstract}

Key words: atrial pacing, alternative sites, atrial fibrillation, Bachmann's bundle

10.17219/acem/61429

\section{Copyright}

Copyright by Author(s)

This is an article distributed under the terms of the

Creative Commons Attribution Non-Commercial License

(http://creativecommons.org/licenses/by-nc-nd/4.0/) 
In the electrical conduction system of the heart, an impulse is generated by the sinoatrial node, located between the superior vena cava ostium and the crista terminalis, and it is propagated down and ahead through the right atrium and simultaneously to the left atrium. ${ }^{1}$ Electrical coupling of both atria is predominantly provided by connections at the level of the Bachmann's bundle and the coronary sinus. The true septum (the fossa ovalis and its limbus) is asynchronous and discordant, usually without contralateral conduction during the sinus rhythm or the atrial pacing. ${ }^{2}$ This course of activation leads to a proper propagation - the $1^{\text {st }}$ part to contract is the right atrium free wall, then the interatrial septum and afterwards the posterior, anterior and lateral walls of the left atrium. An efficient interatrial conduction leads to a physiological delay of the left atrium contraction/interatrial delay which is $24 \pm 21 \mathrm{~ms}$. Thereby, the activation of both atria is represented by a single P-wave in the surface ECG. ${ }^{2}$

\section{Pathophysiology of atrial arrhythmias}

The pathophysiological mechanisms underlying atrial arrhythmia are not yet explicitly understood. It is probably triggered by premature atrial activations, most commonly localized or coming from the region of pulmonary veins or the superior vena cava, and maintained by inhomogeneous atrial conduction velocities and refractory periods. Atrial premature beats can trigger atrial fibrillation in at least 3 ways: an atrial premature contraction after a pause in the sinus rhythm, atrial premature beats with a short coupling interval (the P-on-T phenomenon), and short-long-short atrial sequences. Betts et al. mapped the left atrial endocardial activation during the sinus rhythm and coronary sinus pacing in patients with paroxysmal atrial fibrillation and discovered areas of functional block in the posterior left atrium wall in proximity to the pulmonary veins and suggested that they can serve as a substrate for reentrant arrhythmias, including an atrial flutter and atrial fibrillation. ${ }^{3}$ Bradycardia, atrial dilatation, disturbances of the autonomic nervous system, atrial stretch, and the presence of triggers such as atrial premature contractions are factors which predispose a person to paroxysmal AF.

Atrial fibrillation constitutes the most common arrhythmia in the general population and its incidence doubles with each successive age decade over 50 years, from $0.5 \%$ at age $50-59$ years to almost $9 \%$ at age $80-89$ years. An interesting epidemiological study on atrial fibrillation was conducted by O'Neal et al. ${ }^{4}$ A relatively large group of participants (5226) aged 65 years or over were observed for atrial fibrillation development. The analysis showed that a low heart rate (below 60 beats $/ \mathrm{min}$ ) was associated with an increased risk of this arrhythmia, while no such correlation was observed for a heart rate above
90 beats/min. This could suggest underlying autonomic abnormalities or subclinical sinus node dysfunction.

Atrial fibrillation is associated with atrial remodeling, which may be both the substrate and the consequence of the arrhythmia. Different kinds of remodeling have been described, such as an autonomic neural remodeling, or an electrical and a structural remodeling. Uhm et al. compared effective refractory periods (ERP) in 648 patients with AF and drew the conclusion that patients with persistent AF have shorter ERP compared to those with paroxysmal arrhythmia, probably due to an electrical remodeling of the atria. On the other hand, patients with structural heart disease and sinus node dysfunction have longer ERP than patients with 'lone' AF which is associated with a structural remodeling, ventricular diastolic dysfunction or left atrium enlargement. ${ }^{5}$

\section{The influence of atrial pacing}

As atrial fibrillation is an arrhythmia paroxysmal in nature, the proper assessment of the antiarrhythmic influence of any kind of procedure, device or pharmacological treatment is difficult. Because of a higher prevalence of atrial fibrillation in patients with pacemakers and still an insufficient efficacy of pharmacotherapy in this arrhythmia, the best pacing mode and the best lead location is still being discussed. As the prolonged duration of the atrial activation during pacing, especially from locations changing the physiological pattern of this activation (right atrium lateral wall, right atrium appendage), has a negative impact on both a mechanical and an electrical function of the atria, the impact of atrial pacing site locations on atrial arrhythmogenesis can be observed. So far the study findings on the best pacing site are inconsistent.

The pacing of the atria can eliminate AF triggers such as premature atrial activations and prevent changes in the refractoriness they cause, supporting the atrial synchrony. Atrial pacing not only eliminates bradycardia but also prevents atrial premature contractions and a dispersion of refractoriness which are a substrate for atrial fibrillation. A premature atrial contraction, especially during the vulnerable period of refractoriness, negatively influences atrial myocardium conduction velocity. In a study by Hoffmann et al., 46\% of atrial fibrillation episodes were preceded by atrial premature contractions and $39 \%$ by bradycardia. ${ }^{6}$

\section{The superiority of atrial pacing vs ventricular pacing}

It is now well established that atrial pacing is superior to ventricular pacing for the prevention of atrial fibrillation as it prevents atrial premature beats and atrial bradycardia episodes and helps to avoid an increase of atrial 
pressure associated with atrioventricular dyssynchrony. It is well documented that single lead ventricular pacing, which disrupts the normal atrioventricular synchrony, is associated with a higher incidence of atrial fibrillation than dual chamber pacing (DDD) preserving atrioventricular synchrony. In a study by Andersen et al., patients with sick sinus syndrome (SSS) were observed for 8 years. Atrial pacing was associated with less atrial fibrillation and thromboembolism, higher survival and less heart failure compared to ventricular pacing. ${ }^{7}$ A lower incidence of atrial fibrillation during atrial pacing was also shown in a large group of 1474 patients in the Canadian Trial of Physiological Pacing, however it did not affect the risk of stroke or cardiovascular death. ${ }^{8}$

\section{The influence of atrial pacing on atrial arrhythmogenesis}

Hjortshøj et al. proved in a group of 396 patients that there is no association between the percentage of atrial pacing and the development of AF in patients with sick sinus syndrome, although earlier studies had given different results on this matter. ${ }^{9}$ Elkayam et al. demonstrated that both atrial and ventricular pacing in patients with sick sinus syndrome are associated with a higher risk of AF. A group of 1507 patients was divided into quartiles on the basis of the atrial pacing percentage: The higher this percentage was, the higher the incidence of AF. ${ }^{10} \mathrm{On}$ the other hand, in a study by Inoue et al. in patients with the atrial pacing percentage $(\mathrm{AP} \%)<50 \%$, the AF burden was greater than in those with AP\% > 50\%, which would suggest a protective influence of atrial pacing on atrial fibrillation, however the effect was not always immediate. ${ }^{11}$

According to the results of the DANPACE study, a dual-chamber $\operatorname{DDD}(\mathrm{R})$ pacing with preserved atrioventricular conduction in SSS should be preferred over a single-lead AAIR pacing as the latter is associated with a two-folded risk of reoperation and a higher risk of paroxysmal AF. There was, however, no difference in the overall mortality, chronic atrial fibrillation, heart failure and stroke between those 2 modes of pacing. ${ }^{12}$ Such results are consistent with those of Kuniewicz et al., who also pointed out that tachycardia-bradycardia syndrome and a lack of antiarrhythmic pharmacological therapy with beta-blockers and amiodarone increases the risk of a persistent AF. ${ }^{13}$

An analysis of the data from the DANPACE study showed that AF burden is associated with a longer baseline PQ interval, however neither with the percentage of ventricular pacing nor with the length of programmed AV interval. ${ }^{14}$ Similarly in the MinVPace study, although some algorithms reduced the percentage of ventricular pacing, there was no reduction of atrial fibrillation incidence. ${ }^{15}$ These 2 results can suggest that a longer PQ interval or a greater percentage of ventricular pacing are only indica- tors of a larger scope of conduction abnormalities present also in the atrial muscle, leading to atrial fibrillation.

As already mentioned, different pacing sites vary in electrophysiological properties. A conventional atrial lead location in the right atrium appendage causes a non-physiological activation spreading, resulting in a prolongation of atria activation which can be seen as a broadened $\mathrm{P}$-wave in the surface electrocardiogram.

Bennett used the intracardiac electrophysiological study to assess atrial activation parameters during stimulation at the right atrial appendage, atrial septum, coronary sinus ostium and during a simultaneous stimulation of the right atrial appendage and the ostium of coronary sinus. The atrial activation time was longer during the right atrium appendage pacing compared to other locations. Atrial septum, ostium of coronary sinus and dual site stimulation showed no significant differences. This study suggests potential benefits from intraatrial septum pacing as shortening the activation time of both atria diminishes the dispersion of refractoriness. Dual site pacing showed no additional benefits, which suggests that the septal pacing should be preferred because of the simplicity of the procedure. ${ }^{16}$

Katsivas et al. implanted an atrial lead in the interatrial septum in patients with drug refractory paroxysmal atrial fibrillation and broad P-waves in the surface ECG and proved that this location resulted in shortening P-waves from $118 \pm 10 \mathrm{~ms}$ to $93 \pm 7 \mathrm{~ms} .{ }^{17}$ The interatrial conduction time during pacing was shortened in comparison to sinus rhythm $(115 \pm 18.9 \mathrm{~ms}$ vs $97.8 \pm 10.3 \mathrm{~ms})$.

The manifestation of the atrial activation is a P-wave in the surface electrocardiogram. Lengthening its duration is an easily-measured manifestation of a prolonged atrial activation and is associated with intra- and interatrial conduction disturbances. Prolongation of the P-wave duration is associated with the development of atrial tachyarrhythmias in patients with a pacemaker. ${ }^{18}$ Kirstensen et al. conducted a study in which they assessed atrial parameters in patients with sick sinus syndrome during sinus rhythm and, respectively, 70 and $100 \mathrm{bpm}$ atrial pacing with the lead in the interatrial septum or high right atrium. High right atrium pacing resulted in longer P-waves than pacing with septal location, but during high rate pacing the P-wave was significantly longer than during pacing at $70 \mathrm{bpm}$ in both lead locations. The conclusion was that neither P-wave dispersion nor its duration were predictors of atrial fibrillation paroxysm. ${ }^{19}$ De Sisti et al., however, proved that in patients with sick sinus syndrome after permanent pacemaker implantation, the prolonged $\mathrm{P}$-wave is an independent risk factor of atrial fibrillation. ${ }^{20}$

Advanced interatrial block, presenting in the surface ECG as a P-wave duration over $120 \mathrm{~ms}$ with biphasic +/P-waves in lead II, III and aVF as an expression of a retrograde left atrium activation, is associated with an increased risk of any atrial tachyarrhythmia. ${ }^{21}$ De Sisti et al. 
studied the sinus P-wave morphology, evaluated in leads II and III, in 140 patients with a recurrent paroxysmal AF who received pacemakers. A multivariate analysis showed that drugs before pacing and $\mathrm{P}$-wave duration were independent predictors of $\mathrm{AF}$, and an abnormal P-wave (biphasic $+/$ - or notched $+/+$ ) was the most important predictor of a permanent $\mathrm{AF}^{21}$ Endoh et al. investigated 57 patients with SSS with an atrial lead implanted in the right atrium appendage, who were divided into 2 groups: 23 patients without AF before pacing and 34 with AF before pacing. The latter group was further divided into 2 subgroups: 20 patients with a paced P-wave duration over $130 \mathrm{~ms}$ in leads II and V1, and the remaining 14 patients. The sinus $\mathrm{P}$-wave was significantly longer in the group with a history of AF whilst a recurrence of the arrhythmia was significantly higher in the group with both a history of AF and a longer paced P-wave. This study suggests that a coincidence of SSS with AF is associated with intra-atrial conduction disturbances which may be aggravated by RAA pacing, especially in patients with a prolonged paced $\mathrm{P}$-wave in whom other pacing modalities may be needed to shorten the paced P-wave. ${ }^{22}$

\section{Atrial electrode position}

Stabile et al. studied 47 patients with paroxysmal atrial fibrillation requiring demand pacing who underwent dual chamber pacemaker implantation and an electrophysiological study. ${ }^{23}$ They proved that single site atrial pacing is effective in reducing AF recurrences, however an atrial conduction delay and refractoriness dispersion decrease its efficacy. Pacing at RA sites at which a preferential interatrial conduction exists may pre-excite the left atrium and shorten the total atrial activation time which may result in a reduction of the susceptibility to $\mathrm{AF}^{22}$

\section{Interatrial septum}

De Voogt et al. compared the pacing and sensing properties of leads positioned in the right atrium appendage and the low interatrial septum. The pacing threshold was comparable in both groups. Impedance was higher in the low atrial septum group but the difference was no longer significant after 6 months. Far-field R-wave voltage $>1 \mathrm{mV}$ during ventricular pacing appeared in $87 \%$ of the cases in the right atrium appendage group and in $94 \%$ of the cases in the low atrial septum group, with the difference showing no clinical significance. The time between the spike of a ventricular pacing stimulus and the sensing of the far-field R-wave in the atrium was longer in the RAA group. ${ }^{24}$ The low atrial septum pacing compared to the right atrial appendage pacing had a positive impact on atrial fibrillation prevention. In the study by Minamiguchi et al., after a 1-year follow-up in a group without a history of AF before implantation, 19\% of patients paced in the right atrial appendage developed this arrhythmia while in the group paced in the low atrial septum, only $5.9 \%$ did so. Also, in the group with a positive history of AF, $22 \%$ of the RAA group developed persistent AF, whereas none of the LAS group did. ${ }^{25}$

Hakacova et al. also assessed the properties of septal pacing. They divided patients with conventional indications for pacemaker implantation and recurrent AF into 2 groups: The $1^{\text {st }}$ group had the atrial lead implanted in the atrial septum near the foramen ovale, and the $2^{\text {nd }}$ group received conventional right atrium appendage pacing. The researchers observed no difference in mode-switch episodes and AF burden between the groups, although there was a trend for more AF in the RAA group. A limitation of this study was the small group (44 patients, of whom only 22 completed a 12-month follow-up). Pacing and sensing parameters were comparable in both groups and there were no displacements of electrodes or lead-related complications. ${ }^{26}$ Wang et al. compared low atrial septum pacing to right atrial appendage pacing in patients with sinus node disease and paroxysmal AF and found that septal pacing improved atrial mechanical function and synchronized interatrial contraction. ${ }^{27}$

\section{Bachmann's bundle}

The Bachmann's bundle is a myocardial tract that originates in the crista terminalis, medial to the superior vena cava, in the proximity of the sinoatrial node, which extends over the atrial roof to the left atrium. It is approximately $3 \mathrm{~cm}$ long and in the left atrium it divides into 2 bundles of fibers which distribute to the left atrium. The anterior one advances towards the left atrium appendage, and the posterior one spreads between pulmonary veins. The histological characteristics of the specialized fibers in the Bachmann's bundle resemble those of the Purkinje tissue and they conduct impulses at a higher velocity than the surrounding myocardium, thus being a preferential path for the activation of the left atrium. ${ }^{28}$ The Bachmann's bundle is the fastest way to conduct an impulse between both atria. Disturbances in the conduction result in an impaired electrical and mechanical function of the left atrium which may contribute to the occurrence of atrial fibrillation.

In a study by Bailin et al., a group of 63 patients with the atrial lead implanted in the Bachmann's bundle region was compared to 57 patients with the atrial lead in the right atrium appendage. They observed no differences in pacing and sensing properties during the whole follow-up period, however the P-wave duration in the Bachmann's bundle group was shorter compared to the sinus rhythm whilst in the right atrium appendage group it was longer. After 1 year, patients with the right atrium appendage location had a higher rate of a chronic AF (53\% compared to 
$25 \%)$. Implantation time was comparable in both groups, which indicates the feasibility and a probable efficacy in attenuating the progression of AF. ${ }^{29}$ Bailin et al. also compared the influence of the Bachmann's bundle pacing and the right atrium appendage pacing on the progression to chronic AF in patients with paroxysmal atrial fibrillation without antiarrhythmic pharmacotherapy. The study showed that a significantly greater number of patients in the $\mathrm{BB}$ group maintained the sinus rhythm compared to the right atrium appendage group (75 vs 47\%). The Bachmann's bundle pacing also resulted in a decrease of P-wave duration compared to both RAA pacing and sinus rhythm, while being safe and feasible. ${ }^{30}$

A positive impact of pacing at the Bachmann's bundle region was also proved in studies by our group. This lead location was shown to improve interatrial conduction, and left ventricle filling, as well as to reduce the need for ventricular pacing with all its adverse effects. ${ }^{31,32}$

\section{Multisite pacing}

The concept of multisite pacing, similarly to the Bachmann's bundle pacing, aims to decrease the total atrial activation times.

In the PASTA study, the influence of alternative atrial pacing sites on AF incidence was analyzed. 142 patients were randomized into 4 atrial lead locations: free right atrial wall, right atrium appendage, coronary sinus ostium or dual-site right atrial pacing. ${ }^{33}$ AF episodes were taken into account if the time in AF was over $1 \%$. No statistically significant differences were found in AF occurrence and the percentage of atrial or ventricular pacing after 24 months. The AV delay time was programmed to be 30 ms longer than a spontaneous conduction but the percentage of the ventricular pacing was surprisingly high, with values over $60 \%$, but there were no significant differences between groups. Implantation in the coronary sinus ostium and the dual-site implantation showed significantly longer procedure times and a higher rate of dislodgments of atrial electrodes. Ninety percent of patients in this study were free from AF at the enrollment and only 3-6\% developed this arrhythmia. This was probably a group with an initially low risk of AF and this study shows that in this group of patients, higher rates of dislodgments and longer radioscopy exposure times are not countervailed by antiarrhythmic advantages.

In the study by Saksena et al., 120 patients with at least 2 symptomatic AF episodes in the preceding 3 months and bradyarrhythmia requiring permanent pacing underwent implantation of a dual-chamber pacemaker with 2 atrial leads in the high atrial septum and in the coronary sinus ostium and then were assigned to different pacing modes: high RA overdrive, dual-site right atrial overdrive or support pacing (DDI or VDI) for 6 months each in a randomized sequence. ${ }^{34} \mathrm{AF}$ free survival tended to be longer in the dual-site group, and the risk of a recurrence of arrhythmia was lower in the subgroup of antiarrhythmic drug treated-patients with the dual-site overdrive pacing mode, especially in patients with less than $1 \mathrm{AF}$ event per week. However, a comparative analysis of AF prevention between the pacing modes is greatly impacted by poor adherence and high crossover rates in support and overdrive high RA pacing.

Prakash et al. randomized 79 patients in the same way as Saksena et al. and performed echocardiographic studies which proved that the dual-site atrial pacing improved the atrial filling fraction, whilst the high right atrium pacing resulted in a deterioration of the left ventricle fraction. ${ }^{35}$

Delfaut et al., in their study, proved that in patients with drug-refractory recurrent atrial fibrillation and bradycardia, the atrial pacing in combination with antiarrhythmic drugs eliminates or markedly reduces recurrences of arrhythmia. ${ }^{36}$ The benefit was enhanced by dual-site right atrial pacing as compared to single-site atrial pacing at the high right atrium and the coronary sinus ostium.

Tse et al., in their study, suggested that overdrive pacing at the right atrial appendage and the coronary sinus ostium simultaneously or pacing the interatrial septum leads to a reduction of the atrial activation time and improves mechanical efficiency, which may slow down the progression of a proarrhythmic substrate in the atria. ${ }^{37}$

Biatrial pacing achieved by simultaneous pacing at the coronary sinus and the high right atrium was investigated by D'Allonnes et al. in a group of 86 patients with tachyarrhythmias and a P-wave duration $\geq 120 \mathrm{~ms}$, or an interatrial conduction time $\geq 100 \mathrm{~ms}^{38}$ After 33 months, $64 \%$ of patients maintained the sinus rhythm including $32.6 \%$ without any documented recurrence of arrhythmia. The same location of atrial leads was used in a study by Lau. ${ }^{39}$ In patients with paroxysmal AF without bradycardia, biatrial pacing above the sinus rate prolonged the time to clinical recurrence of arrhythmia compared to high right atrial pacing at 30 beats per minute.

$\mathrm{Yu}$ et al. analyzed the atrial conduction time and electrogram width of the right posterior interatrial septum during drive-train stimulation at the high right atrium, the distal coronary sinus or both sites simultaneously and an early extrastimulation. ${ }^{40}$ Biatrial pacing reduced the atrial conduction delay caused by the early extrastimulation and reduced the increase of electrogram width at the posterior interatrial septum. These effects could possibly prevent induction of atrial fibrillation. Hansen et al. investigated AF susceptibility in mongrel dogs and concluded that multisite pacing simultaneously at the pulmonary vein ostia, left atrium and right atrium decreased atrial activation times and reduced AF induction by $40 \% .{ }^{41}$

Biatrial pacing was compared to right atrial appendage pacing and backup pacing by Levy et al. ${ }^{42}$ In the study, the pacing at a base rate of 70 beats/min showed an antifibrillatory effect compared to an inhibited pacing at 40 beats/ min, but there was no additional benefit from biatrial pacing compared to single site right atrial pacing. 
Lewicka-Nowak et al. described the efficacy and feasibility of atrial resynchronization using multisite atrial pacing at the Bachmann's bundle region and the coronary sinus ostium. ${ }^{43}$ In 97 patients with symptomatic, drug-refractory paroxysmal or persistent atrial fibrillation and a sinus $\mathrm{P}$-wave duration $\geq 120 \mathrm{~ms}$, this treatment resulted in a decrease of the incidence and duration of AF-related hospitalizations, lowering the mean number of antiarrhythmic drugs used and the need for cardioversion. In another study of theirs, in patients with similar characteristics, pacing of the Bachmann's bundle and the coronary sinus ostium had no influence on the global hemodynamics, nevertheless it caused an earlier and more synchronized LA contraction and a reversed right-left contraction sequence. ${ }^{44}$

Despite the encouraging results of some research projects, the usefulness of multisite pacing may be limited by the requirement for 2 atrial leads and a Y connector.

\section{Conclusions}

Atrial pacing is a method of choice in sinoatrial node disease. It provides the physiological electrical activation of the atria and, in the presence of proper atrioventricular conduction, it enables the physiological activation of the ventricles. Historically, the atrial pacing lead was located within the appendix of the right atrium, which was constrained by the lead fixation technique. The introduction of active fixation leads enabled almost all the atrial locations to be paced and the electrical and mechanical properties of such pacing sites are of paramount importance. The overview of the published studies makes it possible to draw a conclusion that the upper and more central locations within the right atrium seem to be superior to the lower and more lateral pacing sites.

The pacing sites incorporating the right atrial roof, the upper part of the interatrial septum and the Bachmann's bundle itself make possible fast interatrial conduction and shorter P-wave duration, resulting in better antiarrhythmic properties. More physiological impulse propagation seems to prevent the long-term remodeling of the atria. Multisite atrial pacing, despite its positive influence on atrial synchrony, does not seem to be widely used due to the adverse side effects caused by numerous intravenous electrodes.

The vast area of uncertainty regarding the long term hemodynamic effects of different atrial pacing sites originates from the lack of large studies with longer follow-up. Atrial arrhythmias may not be a reliable end-point in this regard because other factors influence the arrhythmogenesis to a large extent. There is a need for prospective follow up studies assessing the contribution of different atrial pacing sites to the development of so-called heart failure with preserved systolic function or even simple long-term observations on left atrium volume.

\section{References}

1. Roithinger FX, Cheng J, Sippens-Groenewegen A, et al. Use of electroanatomic mapping to delineate transseptal atrial conduction in humans. Circulation. 1999;100:1791-1797.

2. Lemery R, Soucie L, Martin B, Tang AS, Green M, Healey J. Human study of biatrial electrical coupling: Determinants of endocardial septal activation and conduction over interatrial connections. Circulation. 2004;110:2083-2089.

3. Betts TR, Roberts PR, Morgan JM. High-density mapping of left atrial endocardial activation during sinus rhythm and coronary sinus pacing in patients with paroxysmal atrial fibrillation. J Cardiovasc Electrophysiol. 2004;15:1111-1117.

4. O'Neal WT, Almahmoud MF, Soliman EZ. Resting heart rate and incident atrial fibrillation in the elderly. Pacing Clin Electrophysiol. 2015;38:591-597.

5. Uhm JS, Mun HS, Wi J, et al. Prolonged atrial effective refractory periods in atrial fibrillation patients associated with structural heart disease or sinus node dysfunction compared with lone atrial fibrillation. Pacing Clin Electrophysiol. 2013;36:163-171.

6. Hoffmann E, Sulke N, Edvardsson N, et al. Atrial fibrillation therapy trial investigators. New insights into the initiation of atrial fibrillation: A detailed intraindividual and interindividual analysis of the spontaneous onset of atrial fibrillation using new diagnostic pacemaker features. Circulation. 2006;113:1933-1941.

7. Andersen HR, Nielsen JC, Thomsen PE, et al. Long-term follow-up of patients from a randomised trial of atrial versus ventricular pacing for sick-sinus syndrome. Lancet. 1997;350:1210-1216.

8. Kerr CR, Connolly SJ, Abdollah H, et al. Canadian trial of physiological pacing: Effects of physiological pacing during long-term follow-up. Circulation. 2004;109:357-362.

9. Hjortshøj S, Riahi S, Nielsen JC, Skjøth F, Lundbye-Christensen S, Andersen HR. DANPACE Investigators. Does atrial pacing lead to atrial fibrillation in patients with sick sinus syndrome? Insights from the DANPACE trial. Europace. 2014;16:241-245.

10. Elkayam LU, Koehler JL, Sheldon TJ, Glotzer TV, Rosenthal LS, Lamas GA. The influence of atrial and ventricular pacing on the incidence of atrial fibrillation: A meta-analysis. Pacing Clin Electrophysiol. 2011;34:1593-1599.

11. Inoue $\mathrm{N}$, Ishikawa $\mathrm{T}$, Sumita $\mathrm{S}$, et al. Suppression of atrial fibrillation by atrial pacing. Circ J. 2006;70:1398-1401.

12. Nielsen JC, Thomsen PE, Højberg S, et al. DANPACE Investigators. A comparison of single-lead atrial pacing with dual-chamber pacing in sick sinus syndrome. Eur Heart J. 2011; 32:686-696.

13. Kuniewicz M, Rydlewska A, Karkowski G, Lelakowska-Pieła M, Majewski J, Lelakowski J. Effectiveness of atrial versus atrioventricular pacing for sick sinus syndrome during long-term follow-up. Kardiol Pol. 2015;73:7-16.

14. Nielsen JC, Thomsen PE, Højberg S, et al. DANPACE investigators. Atrial fibrillation in patients with sick sinus syndrome: The association with $\mathrm{PQ}$-interval and percentage of ventricular pacing. Europace. 2012;14:682-689.

15. Veasey RA, Arya A, Freemantle N, et al. The usefulness of minimal ventricular pacing and preventive $\mathrm{AF}$ algorithms in the treatment of PAF: The "MinVPace" study. J Interv Card Electrophysiol. 2010;28: 51-57.

16. Bennett D. Comparison of the acute effects of pacing the atrial septum, right atrial appendage, coronary sinus os, and the latter two sites simultaneously on the duration of atrial activation. Heart. 2000;84:193-196.

17. Katsivas A, Manolis AG, Lazaris E, Vassilopoulos C, Louvros N. Atrial septal pacing to synchronize atrial depolarization in patients with delayed interatrial conduction. Pacing Clin Electrophysiol. 1998;21: 2220-2225.

18. Healey JS, Israel CW, Connolly SJ, et al. Relevance of electrical remodeling in human atrial fibrillation: Results of the asymptomatic atrial fibrillation and stroke evaluation in pacemaker patients and the atrial fibrillation reduction atrial pacing trial mechanisms of atrial fibrillation study. Circ Arrhythm Electrophysiol. 2012;5:626-631.

19. Kristensen L, Nielsen JC, Mortensen PT, et al. Sinus and paced $P$ wave duration and dispersion as predictors of atrial fibrillation after pacemaker implantation inpatients with isolated sick sinus syndrome. Pace. 2004;27:606-614. 
20. De Sisti A, Leclercq JF, Stiubei M, Fiorello P, Halimi F, Attuel P. P Wave duration and morphology predict atrial fibrillation recurrence in patients with sinus node dysfunction and atrial-based pacemaker. PACE. 2002;25,1546-1554.

21. Bayés de Luna $A$, Cladellas $M$, Oter $R$, et al. Interatrial conduction block and retrograde activation of the left atrium and paroxysmal supraventricular tachyarrhythmia. Eur Heart J. 1988;9:1112-1118.

22. Endoh Y, Nakamura A, Suzuki T, et al. Clinical significance of prolonged $P$ wave width after right atrial appendage pacing In sick sinus syndrome. Circ J. 2003;67:485-489.

23. Stabile G, Senatore G, De Simone A, et al. Determinants of efficacy of atrial pacing in preventing atrial fibrillation recurrences. J Cardiovasc Electrophysiol. 1999;10:2-9.

24. de Voogt WG, van Mechelen R, van den Bos A, Scheffer M, van Hemmel NM, Levine PA. Electrical characteristics of low atrial septum pacing compared with right atrial appendage pacing. Europace. 2005;7:60-66.

25. Minamiguchi $H$, Nanto $S$, Onishi $T$, Watanabe $T$, Uematsu $M$ Komuro I. Low atrial septal pacing with dual-chamber pacemakers reduces atrial fibrillation in sick sinus syndrome. J Cardiol. 2011;57:223-230.

26. Hakacova N, Velimirovic D, Margitfalvi P, Hatala R, Buckingham TA. Septal atrial pacing for the prevention of atrial fibrillation. Europace. 2007;9;1124-1128.

27. Wang $M$, Siu CW, Lee $K L$, et al. Effects of right low atrial septal vs right atrial appendage pacing on atrial mechanical function and dyssynchrony in patients with sinus node dysfunction and paroxysmal atrial fibrillation. Europace. 2011;13(9:1268-1274.

28. Savitt MA, Rankin JS. Use of Bachmann's bundle for bipolar atrial pacing. Ann Thorac Surg. 1993;56:183-184.

29. Bailin SJ, Adler S, Giudici M. Prevention of chronic atrial fibrillation by pacing in the region of Bachmann's bundle: Results of a multicenter randomized trial. J Cardiovasc Electrophysiol. 2001;12:912-917.

30. Bailin S. Is Bachmann's bundle the only site for single-site pacing to prevent atrial fibrillation? Results of multicenter randomized trial. Card Electrophysiol Rev. 2003;7:325-328.

31. Sławuta A, Skoczyński P, Kliś M, et al. Bachmann's bundle pacing improves left ventricle filling and reduces the need for ventricular pacing. Eur Heart J. 2014;35:90(Abstract suppl).

32. Sławuta A, Skoczyński P, Bańkowski T, Kliś M, Gajek J. Bachmann's bundle pacing not only improves interatrial conduction but also reduces the need for ventricular pacing. Eur Heart $J$. 2013;34:489(Abstract suppl).

33. Spitzer S, Wacker P, Gazarek S, Malinowski K, Schibgilla V. Primary prevention of atrial fibrillation: Does the atrial lead position influence the incidence of atrial arrhythmias in patients with sinus node dysfunction? Results from PASTA study. PACE. 2009;32:1553-1561.
34. Saksena $S$, Prakash A, Ziegler $P$, et al. Improved suppression of recurrent atrial fibrillation with dual-site right atrial pacing and antiarrhythmic drug therapy. J Am Coll Cardiol. 2002;40:1140-1150.

35. Prakash A, Saksena S, Ziegler PD, et al. Dual site right atrial pacing can improve the impact of standard dual chamber pacing on atrial and ventricular mechanical function in patients with symptomatic atrial fibrillation: Further observations from the dual site atrial pacing for prevention of atrial fibrillation trial. $J$ Interv Card Electrophysiol. 2005;12:177-187.

36. Delfaut P, Saksena S, Prakash A, Krol RB. Long-term outcome of patients with drug refractory atrial flutter and fibrillation after single- and dual-site right atrial pacing for arrhythmia prevention. J Am Coll Cardiol. 1998;32:1900-1908.

37. Tse HF, Hettrick DA, Mehra R, Lau CP. Improved atrial mechanical efficiency during alternate- and multiple-site atrial pacing compared with conventional right atrial appendage pacing: implications for selective site pacing to prevent atrial fibrillation. J Am Coll Cardiol. 2006;47:209-212.

38. D'Allonnes GR, Pavin D, Leclercq C, et al. Long-term effects of biatrial synchronous pacing to prevent drug-refractory atrial tachyarrhythmia: A nine-year experience. J Cardiovasc Electrophysiol. 2000;11:1081-1091.

39. Lau CP, Tse HF, Yu CM, et al. New indication for preventive pacing in atrial fibrillation (NIPP-AF) investigators. Dual-site atrial pacing for atrial fibrillation in patients without bradycardia. J Am Cardiol. 2001;88:371-375.

40. Yu WC, Chen SA, Tai CT, Feng AN, Chang MS. Effects of different atrial pacing modes on atrial electrophysiology: Implicating the mechanism of biatrial pacing in prevention of atrial fibrillation. Circulation. 1997;96:2992-2996.

41. Hansen JC, Latchamsetty R, Lavi N, et al. High-density biatrial pacing protects against atrial fibrillation by synchronizing left atrial tissue. J Interv Card Electrophysiol. 2010;27:81-87.

42. Levy T, Walker S, Rochelle J, Paul V. Evaluation of biatrial pacing, right atrial pacing, and no pacing in patients with drug refractory atrial fibrillation. Am J Cardiol. 1999;84:426-429.

43. Lewicka-Nowak E, Kutarski A, Dabrowska-Kugacka A, Rucinski P, Zagozdzon P, Raczak G. A novel method of multisite atrial pacing, incorporating Bachmann's bundle area and coronary sinus ostium, for electrical atrial resynchronization in patients with recurrent atrial fibrillation. Europace. 2007;9:805-811.

44. Lewicka-Nowak E, Dabrowska-Kugacka A, Rucinski P, Kozlowski D, Raczak G, Kutarski A. Atrial function during different multisite atrial pacing modalities in patients with bradycardia-tachycardia syndrome. Circ J. 2009;73:2029-2035. 